\title{
POSTMORTEM INTERVAL ESTIMATION BY THE METHOD OF WAVELET ANALYSIS OF STOKES-POLARIMETRIC MAPPING DATA OF HUMAN VITREOUS BODY LAYERS
}

DOI: 10.36740/WLek202109117

\author{
Yuliya V. Sarkisova, Viktor T. Bachynskiy, Oleksandr I. Garazdiuk, Ivan V. Garazdiuk, Iana M. Teleki \\ BUKOVINIAN STATE MEDICAL UNIVERSITY, CHERNIVTSI, UKRAINE
}

\begin{abstract}
The aim: To develop a set of forensic criteria for determining PMI on the basis of complex selective statistical data analysis of magnitude distributions of the wavelet-amplitude coefficients of VB polycrystalline films microscopic images.

Materials and methods: The object of study are polycrystalline films of VB, taken from 41 cadavers of both sexes aged from 37 to 79 -year with pre-known time of death coming ranged from 3 to $36 \mathrm{~h}$. Measuring the coordinate allocation meanings of parameters of polarization in the points of microscopic images was carried out at the location of the standard Stokes-polarimeter.

Results: The magnitudes of statistical moments of the 1st-4th orders linearly vary within 36 hours. It was revealed that the data of time changes of the asymmetry and the excess are the most sensitive to necrotic changes in the polycrystalline structure of such samples.

Conclusions: The scale-selective approach provides an increase in the range of sensitivity up to $36 \mathrm{~h}$ and increase the accuracy of the PMl estimation up to $45 \mathrm{~min}$.
\end{abstract}

KEY WORDS: postmortem interval estimation, human vitreous body, Forensic Medicine

Wiad Lek. 2021;74(9 p.l):2118-2122

\section{INTRODUCTION}

Determining the time of death is of great importance in determining the circumstances of the death of a person who died in unknown circumstance. Even more relevant this information is in the autopsy and investigation of the killings [1].

Postmortem interval (PMI) is determined by different indicators in the study of various tissues and organs of the corpse using numerous methods of investigation $[1,2]$. However, the determination of the limitation of death has long been carried out mainly organoleptically with the study of post-mortem processes, the degree of development of early and late cadaveric changes. They are very informative and of great forensic significance, but in some cases they do not allow to reliably answer the questions posed to the expert because of a wide range of variables [3].

At the present stage of science development, laser optical methods are effective for studying structural changes in biological tissues and liquids $[4,5]$. Thus, on the example of brain tissue, muscles, parenchymatous organs, and in the study of liquor films, it was found that the temporal dynamics of changes in the morphological structure of biological tissues and fluids in the post-mortem period is accompanied by dynamic patterns of change in the structure of their polarization images, which is the basis for determining PMI $[6,7]$. The monitoring of vitreous body (VB) biochemical changes has already been successfully used to diagnose PMI $[3,8,9]$, it is appropriate to attempt to investigate its structural changes by polarization methods.

\section{THE AIM}

The aim was to develop a set of forensic criteria for determining PMI on the basis of complex selective statistical data analysis of magnitude distributions of the wavelet-amplitude coefficients of VB polycrystalline films microscopic images.

\section{MATERIALS AND METHODS}

The object of study is polycrystalline films of VB taken from 41 cadavers of both sexes aged from 37 to 79 -year with pre-known time of death (the cause of the death was cardiovascular disease) ranged from 3 to $36 \mathrm{~h}$. Potassium levels were examined in all VB samples for control. Concerning the time that passed after death, the selection is as follows: $3 \mathrm{~h}-5$ cases $(12.2 \%), 6 \mathrm{~h}-7$ cases $(17.1 \%)$, $12 \mathrm{~h}-7$ cases $(17.1 \%), 18 \mathrm{~h}-10$ cases $(24.4 \%), 24 \mathrm{~h}-6$ cases $(14.6 \%), 36 \mathrm{~h}-6$ cases $(14.6 \%)$.

The VB was collected in accordance with source [10]. Potassium level was determined by using an automatic biochemical analyzer.

Coordinate distributions of fourth parameter of the Stokes vector (hereinafter "phase parameter" - PP) VB polycrystalline films microscopic image values were determined for each sample of VB in the optical arrangement of the Stokes polarimeter. Experimental measurements of Stokes-parametric images of biological layers were carried out according to the method presented in [4]. 
Table I. Temporal dynamics of changes in the magnitude of the statistical moments of the 1st -4 th orders $\left(S M_{i=1 ; 2 ; 3 ; 4}\right)$, which characterize the distributions of the linear section $C_{15 ; b}$ of the Stokes wavelet coefficients $W_{a, b}$ map of the VB films microscopic images of deceased with different PMI ( $T$, h.)

\begin{tabular}{cccccccc}
\hline SMi & $\boldsymbol{T = 1}$ & $\boldsymbol{T = 6}$ & $\boldsymbol{T = 1 2}$ & $\boldsymbol{T}=\mathbf{1 8}$ & $\boldsymbol{T = 2 4}$ & $\boldsymbol{T}=\mathbf{3 6}$ \\
\hline$S M_{1}$ & $0,18 \pm 0,07$ & $0,25 \pm 0,011$ & $0,31 \pm 0,013$ & $0,38 \pm 0,015$ & $0,44 \pm 0,018$ & $0,58 \pm 0,024$ \\
\hline$\rho$ & $\rho<0,05$ & $\rho<0,05$ & $\rho<0,05$ & $\rho<0,05$ & $\rho<0,05$ & $\rho<0,05$ \\
\hline$S M_{2}$ & $0,11 \pm 0,005$ & $0,16 \pm 0,007$ & $0,21 \pm 0,009$ & $0,25 \pm 0,011$ & $0,31 \pm 0,014$ & $0,39 \pm 0,016$ \\
\hline$\rho$ & $\rho<0,05$ & $\rho<0,05$ & $\rho<0,05$ & $\rho<0,05$ & $\rho<0,05$ & $\rho<0,05$ \\
\hline$S M_{3}$ & $0,43 \pm 0,019$ & $0,54 \pm 0,024$ & $0,65 \pm 0,031$ & $0,75 \pm 0,034$ & $0,86 \pm 0,041$ & $1,08 \pm 0,051$ \\
\hline$\rho$ & $\rho<0,05$ & $\rho<0,05$ & $\rho<0,05$ & $\rho<0,05$ & $\rho<0,05$ & $\rho<0,05$ \\
\hline$S M_{4}$ & $0,49 \pm 0,021$ & $0,62 \pm 0,028$ & $0,75 \pm 0,034$ & $0,89 \pm 0,041$ & $1,02 \pm 0,049$ & $1,29 \pm 0,058$ \\
\hline$\rho$ & $\rho<0,05$ & $\rho<0,05$ & $\rho<0,05$ & $\rho<0,05$ & $\rho<0,05$ & $\rho<0,05$ \\
\hline
\end{tabular}

Table II. Accuracy $( \pm \Delta T)$ of PMl estimation by wavelet analysis by Stokes polarimetric mapping of VB films

\begin{tabular}{ccccccc}
\hline SMi & $\boldsymbol{T = 1}$ & $\boldsymbol{T = 6}$ & $\boldsymbol{T = 1 2}$ & $\boldsymbol{T = 1 8}$ & $\mathbf{T = 2 4}$ & $\mathbf{T = 3 6}$ \\
\hline$S M_{1}$ & $55 \mathrm{~min}$ & $59 \mathrm{~min}$ & $57 \mathrm{~min}$ & $61 \mathrm{~min}$ & $60 \mathrm{~min}$ & $57 \mathrm{~min}$ \\
\hline$S M_{2}$ & $56 \mathrm{~min}$ & $56 \mathrm{~min}$ & $55 \mathrm{~min}$ & $56 \mathrm{~min}$ & $57 \mathrm{~min}$ & $49 \mathrm{~min}$ \\
\hline$S M_{3}$ & $44 \mathrm{~min}$ & $47 \mathrm{~min}$ & $46 \mathrm{~min}$ & $47 \mathrm{~min}$ & $49 \mathrm{~min}$ \\
\hline$S M_{4}$ & $43 \mathrm{~min}$ & $45 \mathrm{~min}$ & $45 \mathrm{~min}$ & $44 \mathrm{~min}$ & $46 \mathrm{~min}$ & $46 \mathrm{~min}$ \\
\hline
\end{tabular}

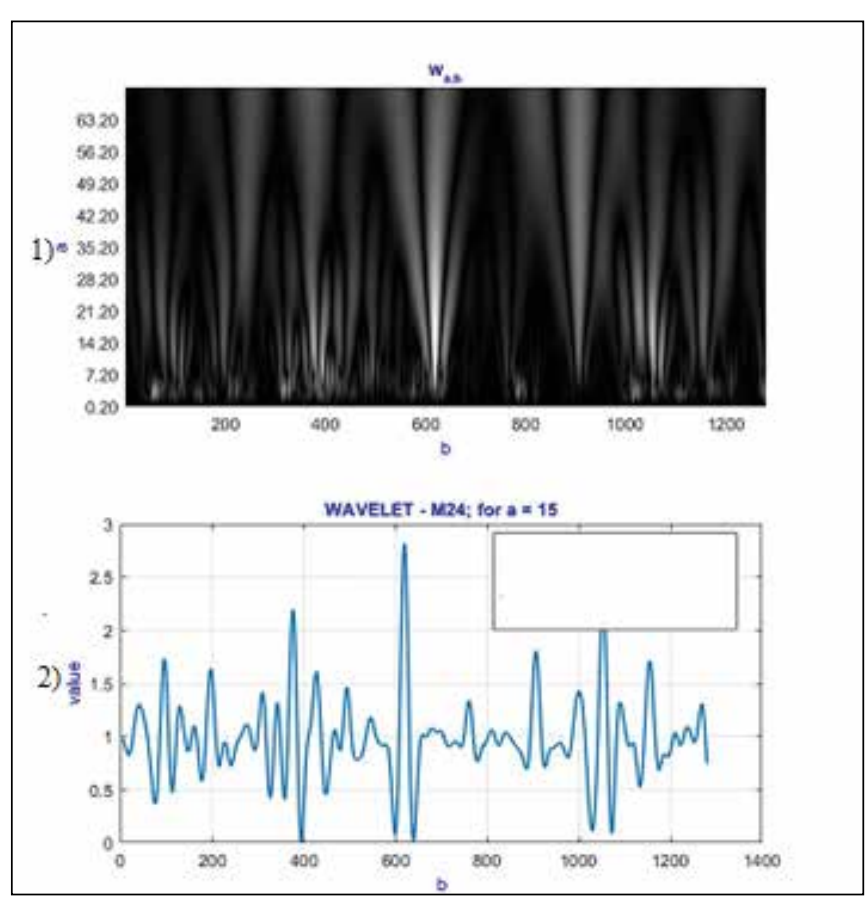

Fig. 1. Maps (1) and linear dependences (2) of the wavelet coefficients amplitude of the VB microscopic image Stokes vector PP for PMI $3 \mathrm{~h}$.

Statistical processing of the calculated values of set of points that characterize the PP distributions was carry out. The depending on the time change of the most sensitive points of statistical values were built.

\section{RESULTS}

We found that the degree and intensity of the increase in the amount of the VB potassium are directly dependent on PMI.

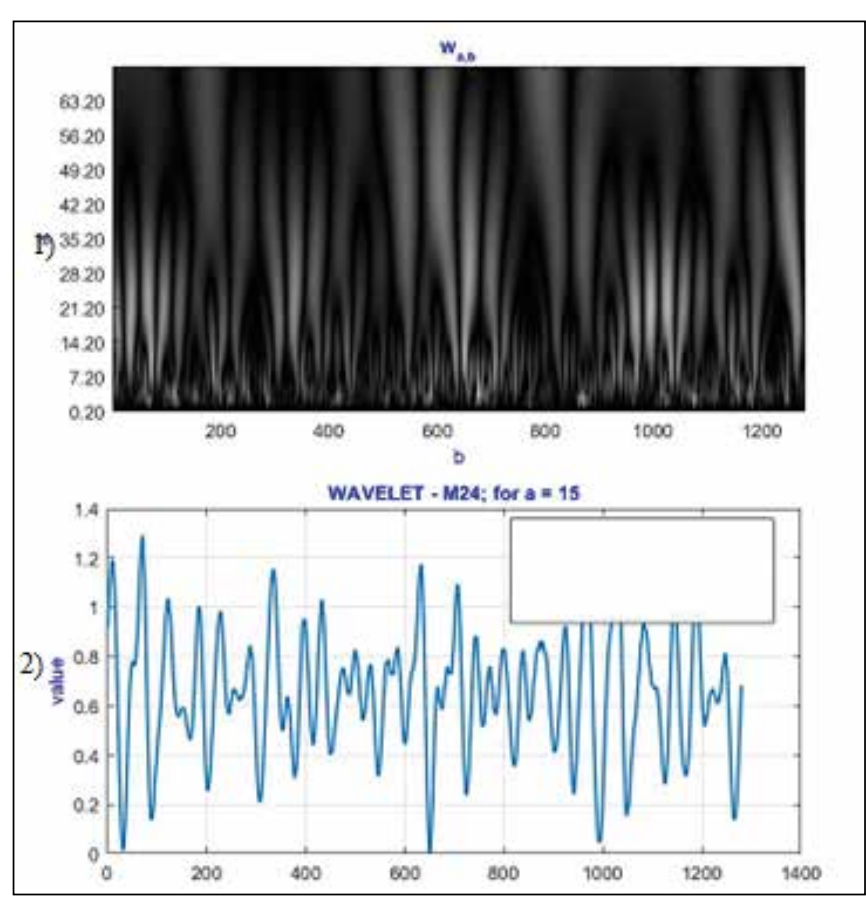

Fig. 2. Maps (1) and linear dependences (2) of the wavelet coefficients amplitude of the VB microscopic image Stokes vector PP for PMI $12 \mathrm{~h}$.

According to the amount of potassium, PMI can be set with an accuracy of 4-6hours in the interval up to 18 hours from the time of death and with an accuracy of 8-12 hours at a later date of PMI.

Necrotic changes in the polycrystalline structure of VB begin with changes in the structure of small-scale protein complexes. Based on this, the maximum differences between the necrotic states of VB specimens with different PMI should be sought at small scale changes in the structure of PP maps of microscopic images. 

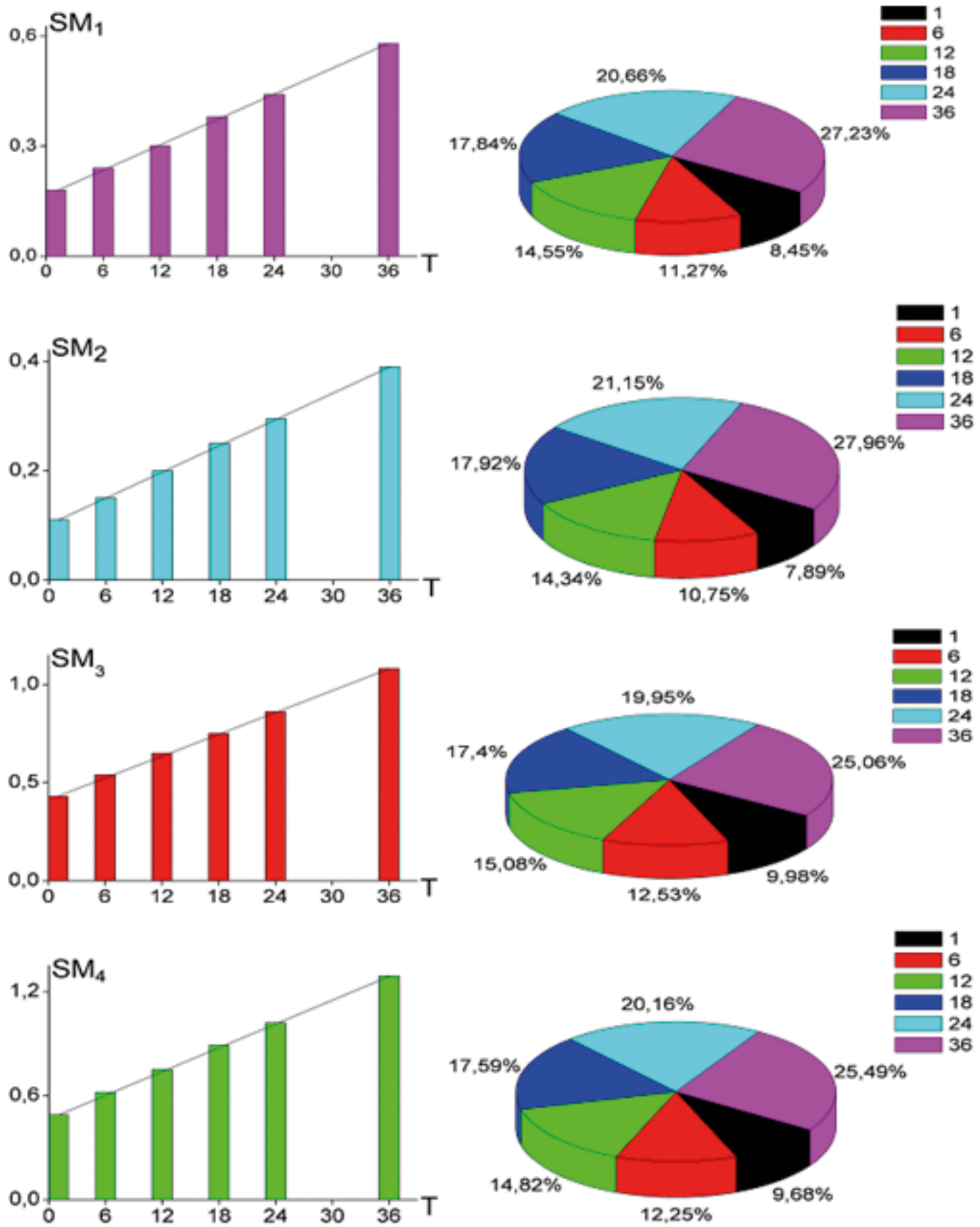

Fig. 3. Time linear and pie charts of changes in the value of the statistical moments of the 1st- 4 th orders $(S M i=1 ; 2 ; 3 ; 4)$, which characterize the distribution of the linear section $C 15, b$ of the wavelet map of the coefficients Wa, b of the Stokes vector of VB films microscopic images from deceased with different PMI (T, h)

In Fig. 1 and Fig. 2 presents the results of studies of the maps of the magnitudes of the amplitude distribution of the wavelet coefficients $K_{a, b}$ (fragments (1)), which characterize the large- and small-scale $(a=15)$ structure (fragments (2)) of the PP coordinate distributions value of microscopic images of optically anisotropic structures of VB layers in case of different PMI (3 hours - Fig. 1) and (12 hours - Fig. 2).

Comparative analysis of the results of the study of the coordinate distributions of the wavelet coefficients $W_{a, b}(x, y)$ amplitudes characterizing the PP maps of VB films samples microscopic images of deaths with different PMI revealed the biggest differences between them at the level of small scales $a_{\min }$ of the wavelet function (Fig. 1 and Fig. 2, $2^{\text {nd }}$ fragments).

Objectively, this illustrates the greater level of modulation of the amplitude of the "small-scale" linear dependencies of the wavelet coefficients $C_{a=15, b}$ of the polarization map of the microscopic image of a layer of VB with PMI $3 \mathrm{~h}$. (Fig. 1, fragment (2)).

Quantitatively this scenario of temporal dynamics of necrotic changes in the scale structure of the coordinate distributions of the Stokes vector 4th parameter of VB 
films microscopic images with different PMI illustrates the magnitudes of the statistical moments of the 1st - 4th orders, which characterize the magnitudes of the wavelet coefficient $C_{a=15, b}$ amplitude (see Tab. I).

It was found:

1) the linear range of change in the magnitude of the statistical moments of the 1 st - 4th orders, which characterize the magnitude distributions of the wavelet coefficients $C_{a=15, b}$ of the deceased by the magnitude of the PMI, is $36 \mathrm{~h}$ (see Tab. I);

2) the mean $S M_{1}$ value of the wavelet coefficients $C_{a=15, b}$ distribution amplitude varies within the range of averages means defined for all groups of samples from 0.18 to 0.58 .

3) the dispersion $S M_{2}$ value of the wavelet coefficients $C_{a=15, b}$ distribution amplitude varies within the range of averages means defined for all groups of samples from 0,11 to 0,39 ;

4) the asymmetry $S M_{3}$ value of the wavelet coefficients $C_{a=15, b}$ distribution amplitude varies within the range of averages means defined for all groups of samples from 0,43 to 1,08 ;

5) the kurtosis $S M_{4}$ value of the wavelet coefficients $C_{a=15, b}$ distribution amplitude varies within the range of averages means defined for all groups of samples from 0,49 to 1,29 .

From the data obtained it can be seen that the magnitudes of all statistical moments of the 1st-4th orders (the mean (1), the dispersion (2), the asymmetry (3) and the kurtosis (4)) linearly vary within 36 hours. At the same time, the most sensitive to necrotic changes in the polycrystalline structure of such samples were the temporal changes of asymmetry $S M_{3}$ and kurtosis $S M_{4}$ (see Fiure 3).

Analysis of the obtained data on the time dependences of the magnitude of the statistical moments of the 1st - 4th orders set, which characterize the magnitudes of the amplitudes of the small-scale structure wavelet coefficients of VB films microscopic images PP coordinate distributions at different intervals of the PMI, revealed the maximum level (highlighted in gray) accuracy in the determination of PMI within 43- 46 min (see Table II).

\section{DISCUSSION}

A large number of scientific works are devoted to the development and improvement of existing methods for determining the PMI. In particular, VB as a subject of research aroused great interest. Many studies are known based on the determination of the electrolyte composition of VB. Patel U.P. and co-authors established the rule of 12: every 12 hours of PMI, potassium concentration in VB increases by approximately $2 \mathrm{mmol} / \mathrm{l}$ [9]. In scientific work, Tumram N.K. and co-authors confirm that a linear increase in the concentration of potassium was observed in the synovial fluid and VB with increasing PMI, and the results of their study were consistent with previous researchers [1]. Foster S.N. and others proposed the use of a regression equation based on the level of potassium in VB, which allows you to set the PMI up to 24 hours [8]. The limitation of this study is that it does not take into account the ambient temperature, which may affect the obtained results. Also known works of other authors, who note that this technique has some drawbacks [3]: potassium levels in VB may be elevated or decreased before death. In this case, it should be expected that such changes cause another starting point and larger changes in the final values. The researchers also noted a greater increase in potassium levels in young subjects compared to the elderly, which may be due to a significant loss of retinal cells with age and a decrease in thickness [3]. Also, in some cases, potassium levels may differ in different types of death. All this encourages scientists to search for new promising techniques.

In recent years, laser polarimetric techniques have been proven to solve many medical and biological problems. These techniques are based on measuring the coordinate distributions of the polarization of laser beams in biological tissues $[4,11]$. Based on the model of generalized optical anisotropy, a two-wave technique of azimuthal-invariant Mueller-matrix reconstruction of optical anisotropy parameters characteristic of biological crystals was developed. [12]. These methods determine the set of statistical moments of 1-4th order, which characterize the distributions of the parameters of the optical anisotropy of biological tissues [13]. These polarization techniques have also been tested to establish PMI. Garazdyuk M.S. and co-authors propose a method of two-dimensional Stokes polarimetric mapping of azimuth polarization distributions of microscopic images of cerebrospinal fluid films in time monitoring of postmortem changes in optical manifestations of polycrystalline networks to determine PMI. Scientists have established the possibility of setting the PMI with an accuracy of 45 minutes at an interval of 30 hours, and an accuracy of 25 minutes at a short time after death (up to 14 hours) [7].

We believe that it is possible to increase the accuracy and extend the PMI detection range by using VB as the object of study, as it has a unique structure that gives it the properties of uniaxial liquid crystals. As a result of large-scale selective Wavelet image analysis of the VB layers, a sensitivity range of 36 hours was established with a PMI setting accuracy of 45 minutes. The obtained results confirm the diagnostic efficiency of this technique in establishing PMI and the prospects for further research in this area.

\section{CONCLUSIONS}

The temporal dynamics of changes in the magnitude of the statistical moments of the 1 st -4 th orders, which characterize the phase parameter distribution of polarizing microscopic images vitreous body films of the deceased with different postmortem interval are investigated.

The efficiency of the wavelet analysis of the phase parameter distributions of polarizing microscopic images vitreous body films of the deceased with different postmortem interval has been demonstrated.

Scale-selective analysis has been shown to increase the sensitivity range up to 36 hours after death and increase the accuracy of postmortem interval estimation to 45 minutes. 


\section{REFERENCES}

1. Tumram N.K., Bardale R.V., Dongre A.P. Postmortem analysis of synovial fluid and vitreous humour for determination of death interval: $A$ comparative study. Forensic Sci Int. 2011;204(1/3):186-90.

2. Koopmanschap D.H., Bayat A.R., Kubat B. et al. The radiodensity of cerebrospinal fluid and vitreous humor as indicator of the time since death. Forensic Sci Med Pathol. 2016;12(3):248-56.

3. Zilg B., Bernard S., Alkass K. et al. A new model for the estimation of time of death from vitreous potassium levels corrected for age and temperature. Forensic Sci Int. 2015;254:158-66.

4. Bachinskyi V., Boychuk T., Ushenko A. Laser polarimetry of biological tissues and fluids. LAP LAMBERT Academic Publishing. 2017, 204.

5. Vanchuliak 0.la. Veivlet-analiz MAP azymutiv poliaryzatsii lazernykh zobrazhen miokarda dlia diahnostyky hostroi koronarnoi nedostatnosti [wavelet analysis of the maps of the polarization azimuths for myocardial laser images with a view of diagnosing acute coronary insufficiency] Buk Med Herald. 2011;15(3):143-6. (In Ukrainian).

6. Sakhnovskiy M.Yu., Olar 0.I., Garazduyk M.S. et al. Correlation structure of Stokes parametric images of polycrystalline films of human biological fluids. Biosensing and Nanomedicine XI. International Society for Optics and Photonics. 2018; 10728:1072800. doi: 10.1117/12.2320512.

7. Garazdyuk M.S., Bachinskyi V.T., Vanchulyak O.Y. et al. Polarization-phase images of liquor polycrystalline films in determining time of death. Appl Opt. 2016;55(12):B67-71.

8. Foster S.N., Smith P.R., Biggs M. et al. Estimation of postmortem interval using vitreous potassium levels in cases of fatal road traffic collision. Arch Med Sadowej Kryminol. 2016;66(2):71-82.

9. Patel U.P., Patel J., Prajapati P., Govekar G. Study to Evaluate of Time Since Death From Potassium Level of Vitreous Humour. National Journal of Medical Research. 2016; 6(3):255-258.

10. Connolly A.J., FinkbeinerW.E., Ursell P.C. Autopsy Pathology: A Manual and Atlas. Medical Laboratory. Studies. 2015; 33-54.

11. Qi J., He H., Lin J. et al. Assessment of tissue polarimetric properties using Stokes polarimetric imaging with circularly polarized illumination. J Biophotonics. 2018;11(4):e201700139. doi: 10.1002/jbio.201700139.
12. Dubolazov A.V., Vanchuliak O.Ya., Garazdiuk M. et al. Polarization-phase tomography of biological fluids polycrystalline structure. Eleventh International Conference on Correlation Optics. International Society for Optics and Photonics. 2013;9066:906620. doi: 10.1117/12.2053861.

13. Ushenko Yu.0., Tomka Yu.Ya., Pridiy 0.G. et al. Wavelet analysis for Mueller matrix images of biological crystal networks. Semiconductor Physics Quantum Electronics \& Optoelectronics. 2009;12(4):391-8.

\section{ORCID and contributionship:}

Yuliya V. Sarkisova: 0000-0001-6095-1957 A,B,D

Viktor T. Bachynskiy: 0000-0002-6955-7507 E,F

Oleksandr I. Garazdiuk: 0000-0002-0430-7558 ${ }^{\mathrm{C}}$

Ivan V. Garazdiuk: 0000-0003-1605-4612 ${ }^{\text {E,F }}$

Iana M. Teleki: 0000-0001-6753-3467 D,E

\section{Conflict of interest:}

The Authors declare no conflict of interest.

\section{CORRESPONDING AUTHOR Yuliya V. Sarkisova \\ Bukovinian State Medical University \\ 2 Teatralnaya sq., 58000 Chernivtsi, Ukraine \\ tel: +380990585229 \\ e-mail: liquiritia.s@gmail.com}

Received: 07.10.2020

Accepted: 02.08 .2021

A - Work concept and design, B - Data collection and analysis, C - Responsibility for statistical analysis,

D-Writing the article, $\mathbf{E}$-Critical review, $\mathbf{F}$ - Final approval of the article 